\title{
Two-Dimensional Harmonic Retrieval in Correlative Noise Based on Genetic Algorithm
}

\author{
Sun-Yong Wu, ${ }^{1,2}$ Gui-Sheng Liao, ${ }^{1}$ and Zhi-Wei Yang ${ }^{1}$ \\ ${ }^{1}$ National Lab of Radar Signal Processing, Xidian University, Xi'an, Shanxi 710071, China \\ ${ }^{2}$ Department of Computational Science and Mathematics, Guilin University of Electronic Technology, Guilin, Guangxi 541004, China
}

Correspondence should be addressed to Sun-Yong Wu, wusunyong121991@163.com and Gui-Sheng Liao, gsliao@xidian.edu.cn

Received 30 December 2009; Revised 13 May 2010; Accepted 16 June 2010

Academic Editor: Ljubiša Stanković

Copyright ( 2010 Sun-Yong Wu et al. This is an open access article distributed under the Creative Commons Attribution License, which permits unrestricted use, distribution, and reproduction in any medium, provided the original work is properly cited.

\begin{abstract}
We propose a niche Genetic algorithm (GA) for the two-dimensional (2D) harmonic retrieval in the presence of correlative zero-mean, multiplicative, and additive noise. Firstly, we introduce a 2D fourth-order time-average moment spectrum which has extremum values at the harmonic frequencies. On this basis, the problem of harmonic retrieval is treated as a problem of finding the extremum values for which the niche GA is resorted. Utilizing the global searching ability of the GA, this method can improve the frequency estimation performance. The effectiveness of the proposed algorithm is demonstrated through computer simulations.
\end{abstract}

\section{Introduction}

2D harmonic retrieval is of interest in signal processing such as sonar, radar, geophysics, and radio astronomy. In the case of additive noise, some high-resolution techniques such as the 2D MUSIC [1], the 2D MEMP [2], and the 2D ESPRIT method [3] have been developed from their 1D versions. Of these algorithms, the ESPIRIT algorithm is more effective as it does not require to search for the peak value in a $2 \mathrm{D}$ space. Different from the above methods considering white noise, in the presence of colored Gaussian noise, Ibrahim and Gharieb $[4,5]$ have presented some methods based on fourth-order cumulants since the cumulants are insensitive to Gaussian noise while they contain frequency and phase information.

Under certain circumstances, the amplitudes of the received harmonic signals are random since they are usually corrupted by multiplicative noise. For example, multiplicative noise is encountered in underwater acoustic applications due to the dispersive medium [6,7], and random amplitude modulation occurs in Doppler-radar signals when the target scintillates or when the point target assumption is no longer valid [8]. Techniques based on cyclic statistics have been proposed to estimate the $2 \mathrm{D}$ harmonic frequencies in multiplicative noise $[9,10]$. However, these methods are based on the assumption that multiplicative and additive noises are mutually independent and mixing.
In practice, the correlation of the multiplicative and additive noise should be considered. In correlative multiplicative and additive noise, $\mathrm{Wu}$ and $\mathrm{Li}$ [11] has studied the problem of the quadratic nonlinear coupling of $2 \mathrm{D}$ harmonics based on 2D third-order time-average moment spectrum. Another two new 2D cyclic statistics are also introduced to estimate the harmonic frequencies in $[12,13]$, respectively. However, both the methods suffer from a resolution limit and no strategy of searching for the extremum values is discussed. In this paper, we propose a new strategy to improve the frequency estimation performance. Since the fourth-order time-average moment spectrum defined in [13] peaks at the harmonic frequencies, the harmonic retrieval can be treated as finding the extremum values in a 2D space. Utilizing the global searching ability, a GA-based algorithm is presented to estimate the frequencies of $2 \mathrm{D}$ harmonic corrupted by correlative multiplicative and additive noise. Simulation examples show that the improved frequency estimation can be achieved.

The organization of the paper is as follows. In Section 2, we introduce a special 2D fourth-order time-average moment spectrum. In Section 3, a GA-based algorithm is proposed to estimate harmonic frequencies. Numerical examples are presented in Section 4, and conclusions are drawn in Section 5. 


\section{2D Harmonic Model}

Consider a discrete-time $L$-component 2D harmonics in multiplicative and additive noise model

$$
x(m, n)=\sum_{l=1}^{L} s_{l}(m, n) \exp \left\{j\left(\omega_{1 l} m+\omega_{2 l} n+\phi_{l}\right)\right\}+v(m, n),
$$

where $m=0,1, \ldots, T_{1}-1, n=0,1, \ldots, T_{2}-1,\left(\omega_{1 l}, \omega_{2 l}\right)$ denotes the $l$ th frequency pair and $\phi_{l}$ represents the $l$ th phase. $s_{l}(m, n)$ and $v(m, n)$ denote multiplicative and additive noise, respectively. In this paper, the following assumptions are given by

$(\mathrm{AS} 1)\left(\omega_{1 l}, \omega_{2 l}\right) \in(0,2 \pi / 3) \times(0,2 \pi / 3), l=1, \ldots, L$, frequency pairs are mutually unequal and not coupled;

(AS2) $\phi_{l}$ s are deterministic constants in $(-\pi, \pi]$;

(AS3) $s_{l}(m, n)$ s and $v(m, n)$ are mutually correlative and stationary stochastic processes with the mean $m_{s_{l}} \triangleq$ $E\left[s_{l}(m, n)\right]=0$ and $m_{v} \triangleq E[v(m, n)]=0 ;$

(AS4) $s_{l}(m, n)$ s and $v(m, n)$ satisfy the so-called crossmixing condition [12]:

$$
\begin{gathered}
\sum_{\xi_{1} \ldots \xi_{p}=-\infty}^{\infty} \sum_{\eta_{1} \ldots \eta_{p}=-\infty}^{\infty} \sup _{m, n} \mid \operatorname{cum}\left\{s_{l 0}(m, n), s_{l 1}\left(m+\xi_{1}, n+\eta_{1}\right),\right. \\
\left.\ldots, s_{l p}\left(m+\xi_{p}, n+\eta_{p}\right)\right\} \mid \\
<+\infty,
\end{gathered}
$$

where $s_{l i}(m, n) \in\left\{s_{l}(m, n), s_{k}^{*}(m, n)\right\}, s_{0}(m, n) \triangleq$ $v(m, n), i=0, \ldots, p ; l, k=0, \ldots, L$.

Note that if the variance $\sigma_{s_{l}}^{2} \triangleq E\left[s_{l}^{2}(m, n)\right]-m_{s_{l}}^{2}=$ 0 , the model in (1) would become the classical case of harmonics with constant amplitude in additive noise. The first assumption ensures that the fourth-order time-average moment spectrum which will be defined latter peaks at the harmonic frequencies. The second assumption ensures the identifiability of harmonic phases. In the third assumption, without loss of generality, the mean of the additive noise can be assumed to be zero. A nonzero mean can always be estimated consistently via the sample mean and then subtracted from the data. When the mean of multiplicative noise $s_{l}(m, n), m_{s_{l}} \neq 0, \forall l$, Wang et al. [9] and Yang and Li [10] have presented some effective methods to estimate the harmonic frequencies.

Generally, $s_{l}(m, n)$ and $v(m, n)$ are always assumed to be mixing and mutually independent [6-10]. In order to describe the correlativity of multiplicative and additive noise, $\mathrm{Xu}$ et al. [12] derived the cross mixing condition. If $s_{l}(m, n)$ and $v(m, n)$ are mixing and mutually independent, they are also cross mixing. On the contrary, if $s_{l}(m, n)$ and $v(m, n)$ are cross mixing, they must be mixing, but it doesn't mean that they are mutually independent. The correlativity of multiplicative and additive noise can be described by crossmixing condition. The cross-mixing property implies that samples of the processes that are well separated in time can be regarded as approximately independent [14]. Under the cross-mixing condition, time averages of the cyclic statistics converge in the mean-square sense to their sample averages. In model $(1)$, if $s_{l}(m, n)$ and $v(m, n)$ are cross-mixing, the observed signal $x(m, n)$ is cyclostationary, and the sample estimates of its cyclic statistics converge in the sense of mean square and are asymptotically normal [15].

For notational simplicity, let $s_{0}(m, n)=v(m, n)$, $\left(\omega_{10}, \omega_{20}\right)=(0,0), \phi_{0}=0$. Then $(1)$ is given by

$$
x(m, n)=\sum_{l=0}^{L} s_{l}(m, n) \exp \left[j\left(\omega_{1 l} m+\omega_{2 l} n+\phi_{l}\right)\right] .
$$

Definition 1. The special fourth-order time-average moment of $x(m, n)$ is defined as

$$
\begin{aligned}
& \bar{m}_{4 x}(\underline{\tau}, \underline{\xi}) \\
& \triangleq \lim _{T_{1}, T_{2} \rightarrow \infty} \frac{1}{T_{1} T_{2}} \sum_{m=0}^{T_{1}-1} \sum_{n=0}^{T_{2}-1} m_{4 x}(m, n ; \underline{\tau}, \underline{\xi}) \\
& =\lim _{T_{1}, T_{2} \rightarrow \infty} \frac{1}{T_{1} T_{2}} \sum_{m=0}^{T_{1}-1} \sum_{n=0}^{T_{2}-1} E\left\{\left(x^{*}(m, n)\right)^{2} x^{*}(m+\tau, n+\xi)\right. \\
& \times x(m+\tau, n+\xi)\},
\end{aligned}
$$

where $\underline{\tau}=(0, \tau, \tau), \underline{\xi}=(0, \xi, \xi)$.

Definition 2. The special fourth-order time-average moment spectrum is defined as

$$
\begin{aligned}
& \bar{M}_{4 x}(\alpha, \beta) \\
& \triangleq \lim _{T_{1}, T_{2} \rightarrow \infty} \frac{1}{T_{1} T_{2}} \sum_{\tau=0}^{T_{1}-1} \sum_{\xi=0}^{T_{2}-1} \bar{m}_{4 x}(\underline{\tau}, \underline{\xi}) e^{-j \alpha \tau} e^{-j \beta \xi} \\
& =\lim _{T_{1}, T_{2} \rightarrow \infty} \frac{1}{T_{1}^{2} T_{2}^{2}} \\
& \quad \times \sum_{\tau=0}^{T_{1}-1} \sum_{\xi=0}^{T_{2}-1} \sum_{m=0}^{T_{1}-1} \sum_{n=0}^{T_{2}-1} E\left\{\left(x^{*}(m, n)\right)^{2} x^{*}(m+\tau, n+\xi)\right. \\
& \quad \times x(m+\tau, n+\xi)\} e^{-j \alpha \tau} e^{-j \beta \xi} .
\end{aligned}
$$


Theorem 1. From [13], the fourth-order time-average moment spectrum of $x(m, n)$ corresponding to (5) can be obtained by

$$
\begin{aligned}
\bar{M}_{4 x}(\alpha, \beta)=2 \sum_{l_{1}=1}^{L} E\left\{s_{0}^{*}(m, n) s_{l_{1}}^{*}(m, n)\right\} E\left\{s_{0}^{*}(m, n) s_{l_{1}}(m, n)\right\} \\
\times \delta\left(\alpha-\omega_{1 l_{1}}\right) \delta\left(\beta-\omega_{2 l_{1}}\right) \\
+\left[\sum_{l_{2}=1}^{L} E\left\{s_{0}^{*}(m, n) s_{0}^{*}(m, n)\right\}\right. \\
\times E\left\{s_{l_{2}}^{*}(m, n) s_{l_{2}}(m, n)\right\} \\
+E\left\{s_{0}^{*}(m, n) s_{0}^{*}(m, n)\right\} \\
\left.\times E\left\{s_{0}^{*}(m, n) s_{0}(m, n)\right\}\right] \delta(\alpha) \delta(\beta)
\end{aligned}
$$

where $\delta(\alpha)$ is Kronecker function. Equation (6) contains harmonic frequency information. This relation will be proved in the appendix.

From (6), $\bar{M}_{4 x}(\alpha, \beta)$ is unequal to zero only if $(\alpha, \beta)=$ $\left(\omega_{1 l}, \omega_{2 l}\right), l=1, \ldots, L$, or $(\alpha, \beta)=(0,0)$. According to Assumption $1,\left(\omega_{1 l}, \omega_{2 l}\right) \neq(0,0)$, the number of the obtained greatest maxima of $\left|\bar{M}_{4 x}(\alpha, \beta)\right|$ should be $L$ rather than $L+1$ in the $(\alpha, \beta)$ plane.

From [16], $\bar{M}_{4 x}(\alpha, \beta)$ can be estimated using single record, namely,

$$
\begin{aligned}
& \widehat{\bar{M}}_{4 x}(\alpha, \beta) \\
& \triangleq \frac{1}{T_{1}^{2} T_{2}^{2}} \sum_{\tau=0}^{T_{1}-1} \sum_{\xi=0}^{T_{2}-1} \sum_{m=0}^{T_{1}-1} \sum_{n=0}^{T_{2}-1}\{ \\
& \quad \\
& \times x(m+\tau, n+\xi)\} e^{-j \alpha \tau} e^{-j \beta \xi} .
\end{aligned}
$$

Further, we have

$$
\widehat{\bar{M}}_{4 x}(\alpha, \beta) \stackrel{\text { a.s. }}{\longrightarrow} \bar{M}_{4 x}(\alpha, \beta),
$$

where a.s. represents almost sure convergence which is uniform in $\alpha$ and $\beta$. Equation (8) means that the single record estimator $\widehat{M}_{4 x}(\alpha, \beta)$ is strongly consistent. Alternatively, we can estimate the harmonic frequencies by searching for $L$ greatest maxima of $\left|\widehat{M}_{4 x}(\alpha, \beta)\right|$ in the $(\alpha, \beta)$ plane. Consequently, the frequency estimation of $2 \mathrm{D}$ harmonics can be treated as finding the extremum values of binary function. In this paper, the GA is proposed to seek $L$ local optimum solutions.

\section{Genetic Algorithm Realization}

GA is nowadays one of the most popular stochastic optimization techniques which is inspired by natural genetics and biological evolutionary process. It is supposed that individuals with more adaptability in current generation would have better capability of survival and breeding in the next generation. One of the most important advantages of GA is that it can make use of the limited searching processes to automatically find the optimal or near-optimal result in the solution space. Compared with the classical optimization algorithms, GA has such features as follows [17].

(1) GA starts from multipoints instead of one point. Thereby, it could effectively prevent the searching processes from stopping in local optimum solutions.

(2) The optimization rule of GA is varied. It is determined by probability.

(3) The fitness is calculated only from object function. No other information is necessary.

(4) It automatically seeks the optimal result in the whole solution space.

(5) The calculation is relatively simple.

GA evaluates a population and generates a new one iteratively, with each successive population referred to as a generation. Assume the current generation $t$ is $G(t)$. After applying a set of genetic operations, the GA generates a new generation $G(t+1)$ based on the previous generation. Three basic operators are used to manipulate the genetic composition of a population: selection, crossover, and mutation [17].

The detailed implementation of the proposed method is given as follows.

$1^{\circ}$ Fitness Function. In order to perform GA, it is very important to define an appropriate fitness function. In GA, the probability of individual survival to the next generation depends on its fitness value. The greater the fitness value of an individual, the greater probability it has to inherit to the next generation. In this paper, the fitness function is constructed by the operation of getting the absolute value on the nonlinear function (7)

$$
\begin{aligned}
& \text { fitness }(\alpha, \beta) \\
& \begin{array}{r}
=\left|\widehat{M}_{4 x}(\alpha, \beta)\right| \\
=\mid \frac{1}{T_{1}^{2} T_{2}^{2}} \sum_{\tau=0}^{T_{1}-1} \sum_{\xi=0}^{T_{2}-1} \sum_{m=0}^{T_{1}-1} \sum_{n=0}^{T_{2}-1}\left\{\left(x^{*}(m, n)\right)^{2} x^{*}(m+\tau, n+\xi)\right. \\
\quad \times x(m+\tau, n+\xi)\} e^{-j \alpha \tau} e^{-j \beta \xi} \mid,
\end{array}
\end{aligned}
$$

where $x(m, n)$ is the observed signal.

$2^{\circ}$ Generation of the Initial Group. Commonly in GA, the initial population is randomly generated. The real-coded GA is adopted in this paper. The length of chromosome graph is set as 3 . The first two genes denote the frequency pairs and the third gene denotes the fitness value.

The performance of GA is influenced heavily by the population size. GA may run the risk of serious undercovering of the solution space and result in a local optimum 


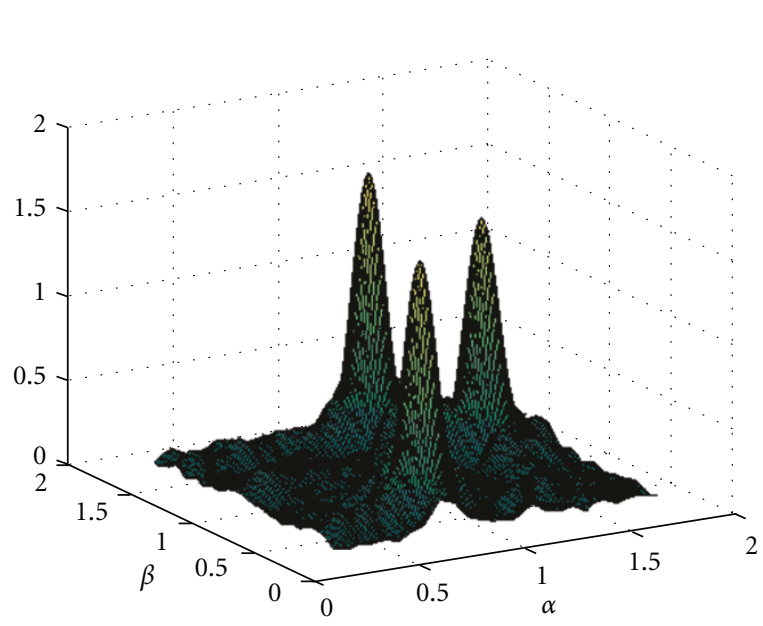

(a) $3 \mathrm{D}$ view

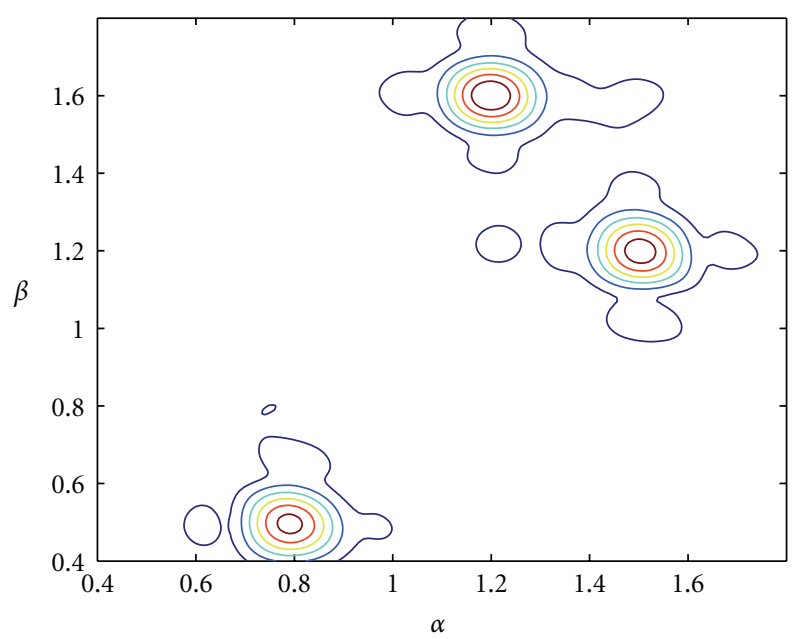

(b) Vertical view

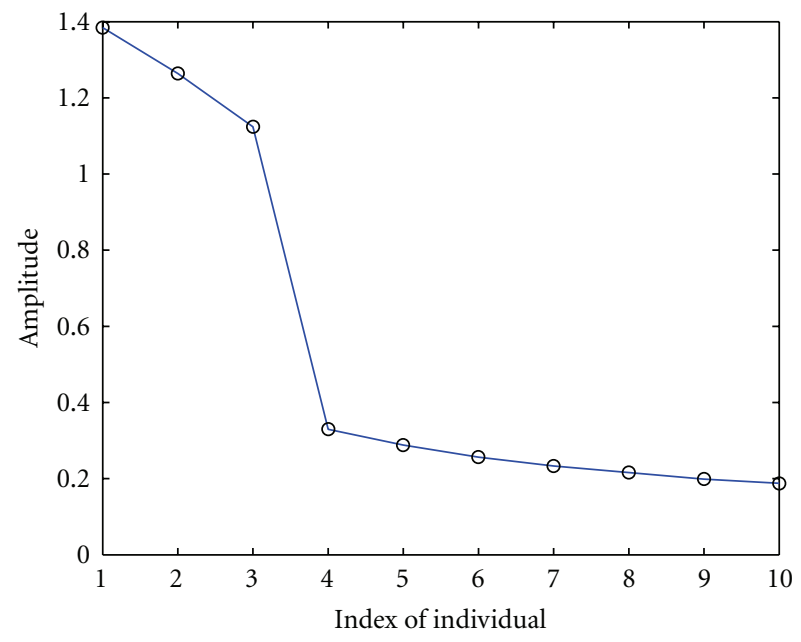

(c) Estimation based on GA

FIGURE 1: $\left|\widehat{M}_{4 x}(\alpha, \beta)\right|$ of $(7), e(m, n)$ is white Gaussian noise, SNR $=0 \mathrm{~dB}$.

when the population size is relatively small, whereas GA would increase the computational load when the population size is relatively large. Consequently, the population size should be chosen according to the problem scale.

$3^{\circ}$ Selection. The operation is to choose the good individual and get rid of the bad one from the group. The larger the fitness, the larger probability the individual has to be selected. To achieve this, the roulette wheel selection is used. The fitness of the $i$ th individual is denoted as $F_{i}$. The selection probability of the $i$ th individual is computed as $P_{i s}=$ $F_{i} / \sum_{i=1}^{m} F_{i}$. At the same time, we reserve the best individual to the next population.

$4^{\circ}$ Crossover. The crossover is to exchange some parts of an individual with corresponding parts of another. The crossover is performed in the following way. Assume that $X_{i}$ and $X_{j}$ are pairs of parent chromosome, whether to crossover or not depends on the crossover probability $P_{c}$. The result of crossover is

$$
\begin{aligned}
X_{i}^{\prime} & =(1-\lambda) X_{i}+\lambda X_{j}, \\
X_{j}^{\prime} & =(1-\lambda) X_{j}+\lambda X_{i},
\end{aligned}
$$

where $\lambda$ is a uniformly distributed random number in $[0,1]$.

$5^{\circ}$ Mutation. The mutation operator adopts "Nonuniform Mutation" [18]. Compared with the classical uniform mutation operator, this operator has the advantage of making fewer changes on the genes with the number of generations increasing. This property makes the tradeoff between exploration and exploitation. It is more favorable to have exploration in the early stages of the algorithm, while exploitation becomes of greater importance when the obtained solution is closer to the optimal solution. The mutation can be completed in the following way: assuming $x_{k}$ is the $k$ th component of the individual $X_{i}$, the mutation 


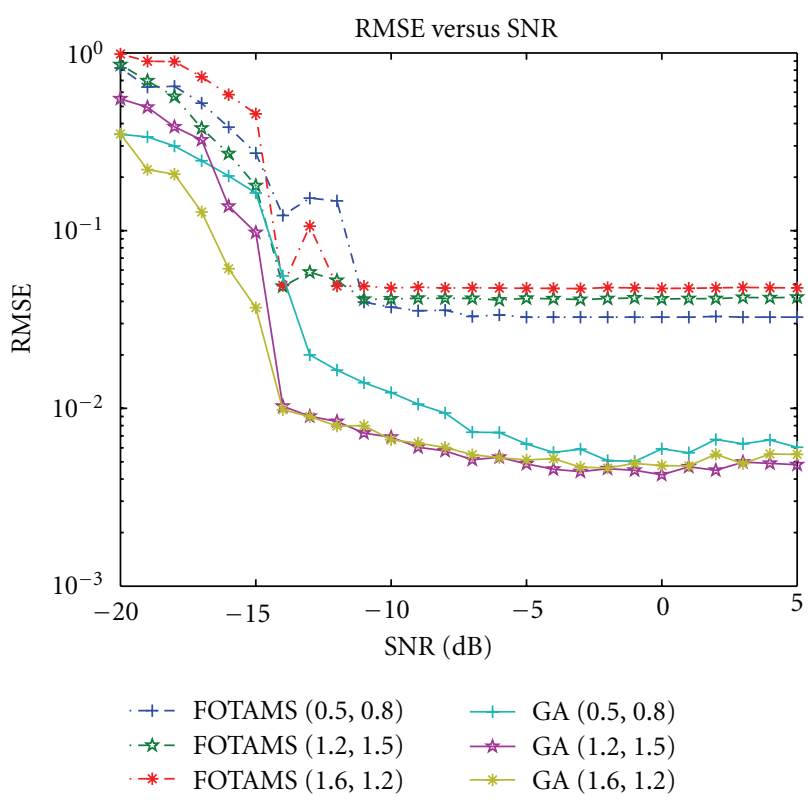

FIGURE 2: RMSEs of frequency estimation versus the SNR, $e(m, n)$ is white Gaussian noise, the data size is $50 \times 50$.

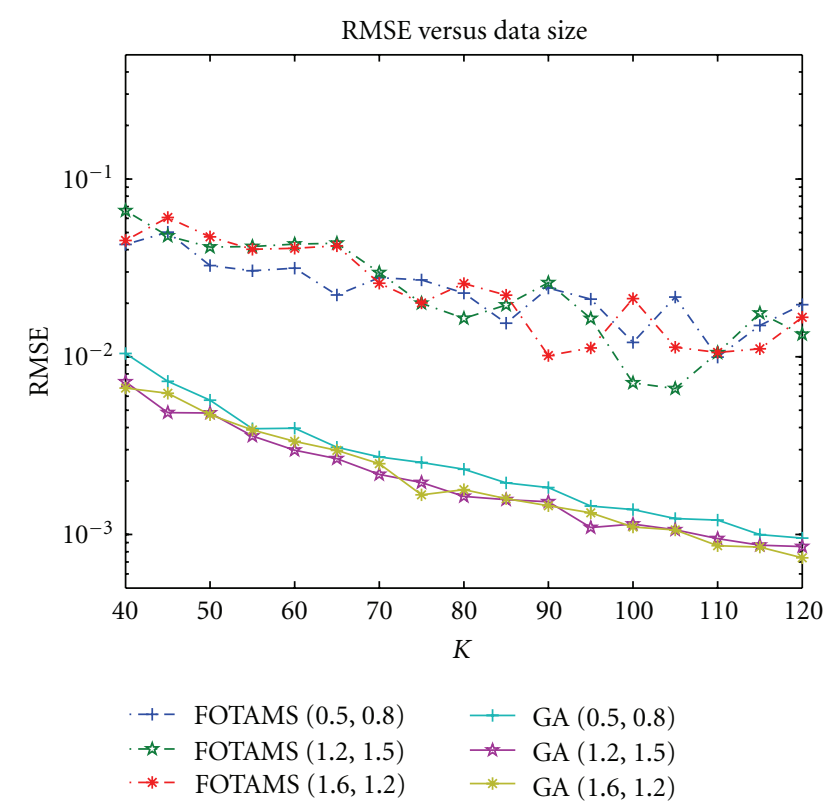

FIGURE 3: RMSEs of frequency estimation versus the data size, $e(m, n)$ is white Gaussian noise, $\mathrm{SNR}=0 \mathrm{~dB}$.

probability $P_{m}$ determines whether to mutate or not. The result of mutation is

$$
\begin{gathered}
x_{k}^{\prime}= \begin{cases}x_{k}+\Delta\left(x, u_{\max }^{k}-x_{k}\right), & \text { if } \beta>0.5, \\
x_{k}-\Delta\left(x, x_{k}-u_{\min }^{k}\right), & \text { if } \beta<0.5,\end{cases} \\
\Delta(x, y)=y \cdot\left(1-r^{(1-t / T) b}\right),
\end{gathered}
$$

where positive number $b$ controls the dependence degree of random fluctuation to evolution number $t . r$ and $\beta$ are uniformly distributed as random numbers on the interval $[0,1] . T$ is the largest evolution number.

$6^{\circ}$ Condition to Terminate the GA Iterations. When the number of generation reaches $T$, the iterations would be terminated.

In this paper, we seek all the optimal solutions of nonlinear function $\left|\widehat{M}_{4 x}(\alpha, \beta)\right|$, including local optimal solutions and global optimal solutions. However, the simple genetic algorithm is unable to get all optimal solutions. According to the niche phenomenon in nature $[19,20]$, a niche GAbased method is proposed to estimate harmonic frequencies. A niche in nature can be viewed as a subspace in the environment. Accordingly, a niche is commonly thought as a peak of the fitness function. The niche techniques gather the individuals on several peaks of fitness function in the population according to genetic likeness. The structure of a niche is implemented by decreasing the fitness value of the individual. The concrete method is implemented by calculating the Euclidean distance between parent individual and arbitrary other child individual and then judging whether two individuals are in the circle defined by estimating niche radius $d$. Compare with simple GA, niche GA can find more than one optima during evolution. The basic steps of the algorithm are given as follows.

(1) Set the generation number $t=0$. Create an initial population which includes $M$ individuals and evaluate their fitness.

(2) Sort the population according to their fitness and memorize the first $N$ individuals.

(3) Produce a new population through selection, crossover and mutation.

(4) Evaluate the fitness of every new individual.

(5) Keep the $M$ individuals from step 3 and $N$ memorial individuals from step 2. Evaluate the distance $d$ between each two of them. Introduce a penalty $P$ to the individual with lower fitness when $d<R$.

(6) Combine the $M$ individuals with the $N$ individuals. Sort the $M+N$ individuals according to their fitness. Save the first $N$ individuals and the first $M$ individuals.

(7) Set $t=t+1$ and repeat steps 3-6 until $t=T$.

\section{Simulations}

Computer simulations are presented here to illustrate the main aspects of this paper. In all simulations, we generate 


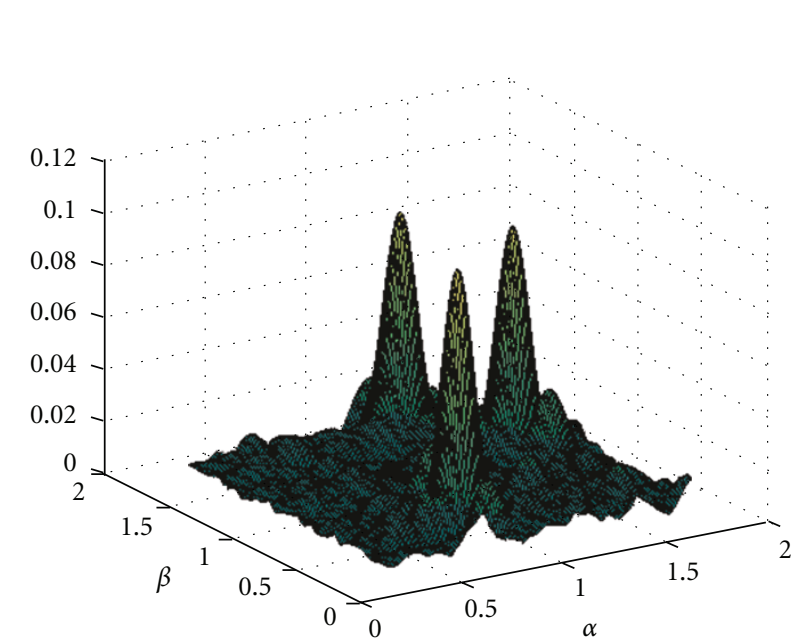

(a) 3D view

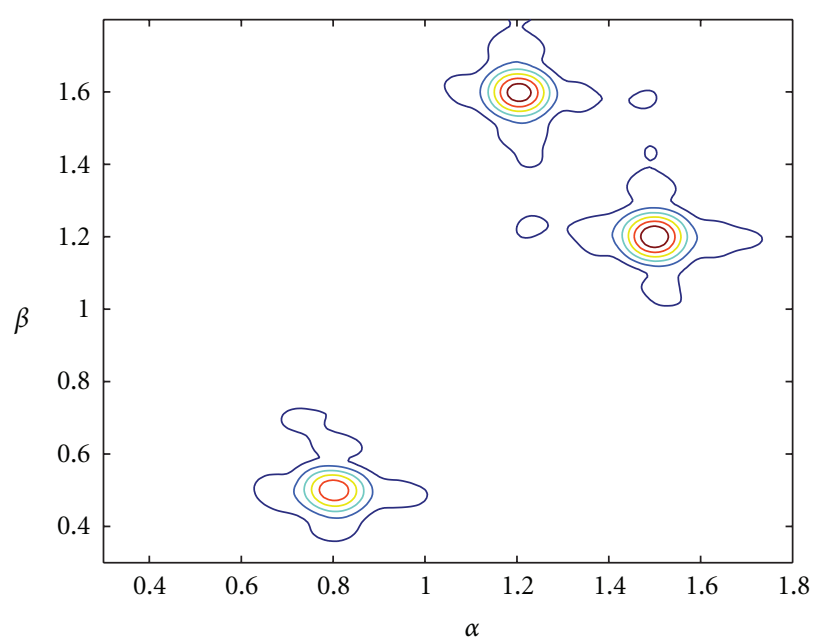

(b) Vertical view

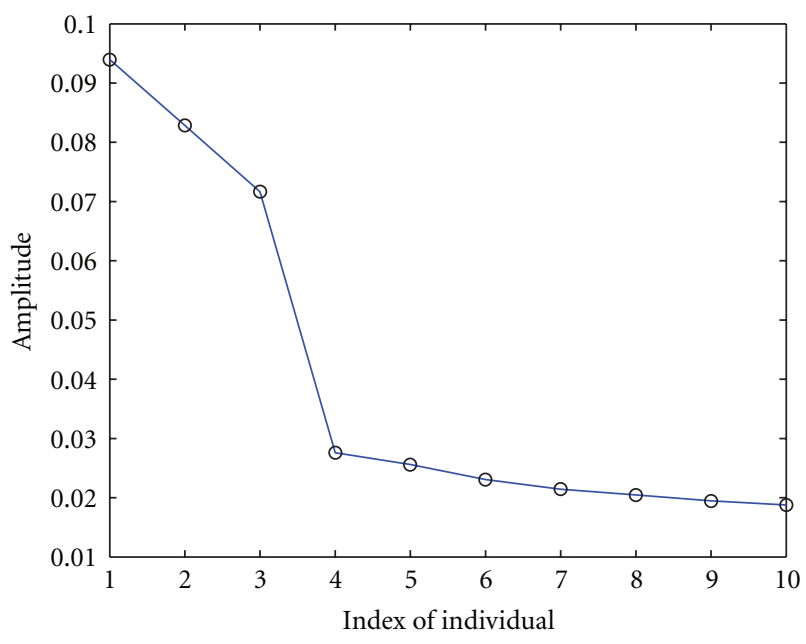

(c) Estimation based on GA

FigURE 4: $\left|\widehat{M}_{4 x}(\alpha, \beta)\right|$ of $(7), e(m, n)$ is i.i.d white exponential noise, $\mathrm{SNR}=0 \mathrm{~dB}$.

(1) with $L=3,\left(\omega_{11}, \omega_{21}\right)=(0.5,0.8),\left(\omega_{12}, \omega_{22}\right)=$ $(1.2,1.5),\left(\omega_{13}, \omega_{23}\right)=(1.6,1.2), \phi_{1}=0.1, \phi_{2}=0.2$, and $\phi_{3}=0.6$. The multiplicative noises are generated by

$$
\begin{aligned}
s_{1}(m, n)= & e(m, n)-0.5 e(m-1, n-1) \\
& -0.5 e(m-2, n-2), \\
s_{2}(m, n)= & e(m, n)-0.3 e(m-1, n-1) \\
& -0.7 e(m-2, n-2), \\
s_{3}(m, n)= & e(m, n)-0.4 e(m-1, n-1) \\
& -0.6 e(m-2, n-2),
\end{aligned}
$$

where the mean $m_{s_{l}}=0, l=1,2,3$. The additive noise $v(m, n)$ is also generated by $e(m, n)$ with the mean $m_{v}=0$. The signal-to-noise ratio (SNR) is defined as

$$
\mathrm{SNR}=10 \log _{10}\left[\frac{\sum_{l=1}^{L}\left(\sigma_{s_{l}}^{2}\right)}{\sigma_{v}^{2}}\right] .
$$

Example 1. Consider $e(m, n)$ as white Gaussian noise with the mean $m_{e}=0$ and the variance $\sigma_{e}^{2}=1$. The fourth-order time-average moment spectrum is computed according to (7). Figure 1 shows $\left|\widehat{M}_{4 x}(\alpha, \beta)\right|$ when the SNR is $0 \mathrm{~dB}$ and the data size is $50 \times 50$. $\left|\widehat{M}_{4 x}(\alpha, \beta)\right|$ which varies with $\alpha$ and $\beta$ is plotted in Figure 1(a). It can be observed that there are three obvious peaks. It is shown in Figure 1(b) that three peaks locate at the accurate positions $\left(\omega_{1 l}, \omega_{2 l}\right), l=1,2$, and 3. Figure 1(c) shows the ten greatest estimated mean of $\left|\widehat{M}_{4 x}(\alpha, \beta)\right|$ using the GA from 100 Monte Carlo runs. The $x$-coordinate denotes the index of individuals and the $y$-coordinate denotes the corresponding estimated mean. It can be shown that there is an obvious boundary among the estimated values. The estimated number of harmonics is 3 . However, if the SNR is very low, there is no obvious boundary among the estimate values. In this situation, we should estimate the number of harmonics firstly. More detail of estimating the number of harmonics has been presented in $[21,22]$. 


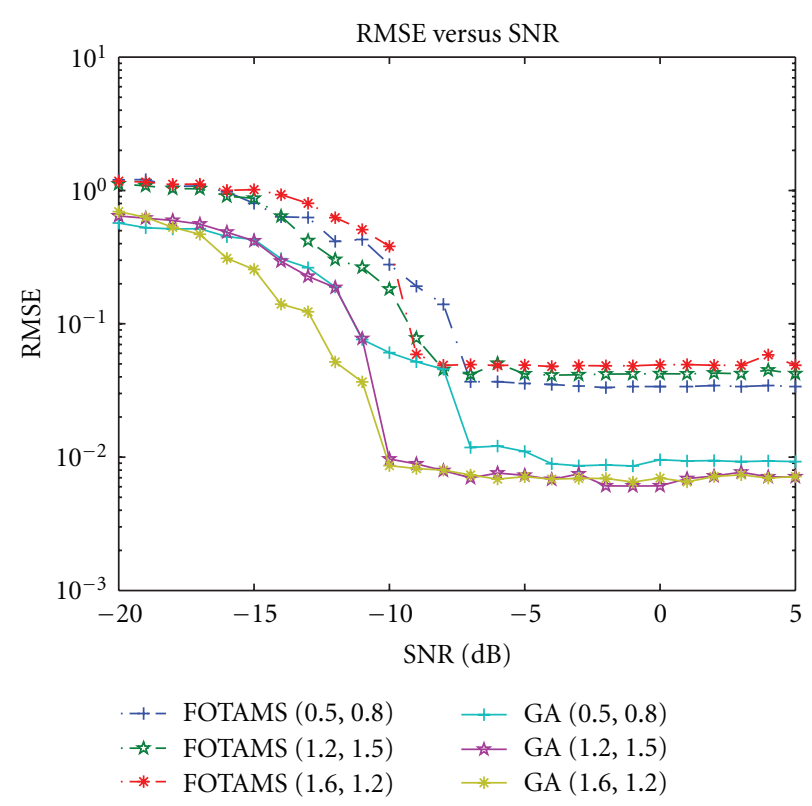

FIGURE 5: RMSEs of frequency estimation versus the SNR, $e(m, n)$ is i.i.d white exponential noise, the data size is $50 \times 50$.

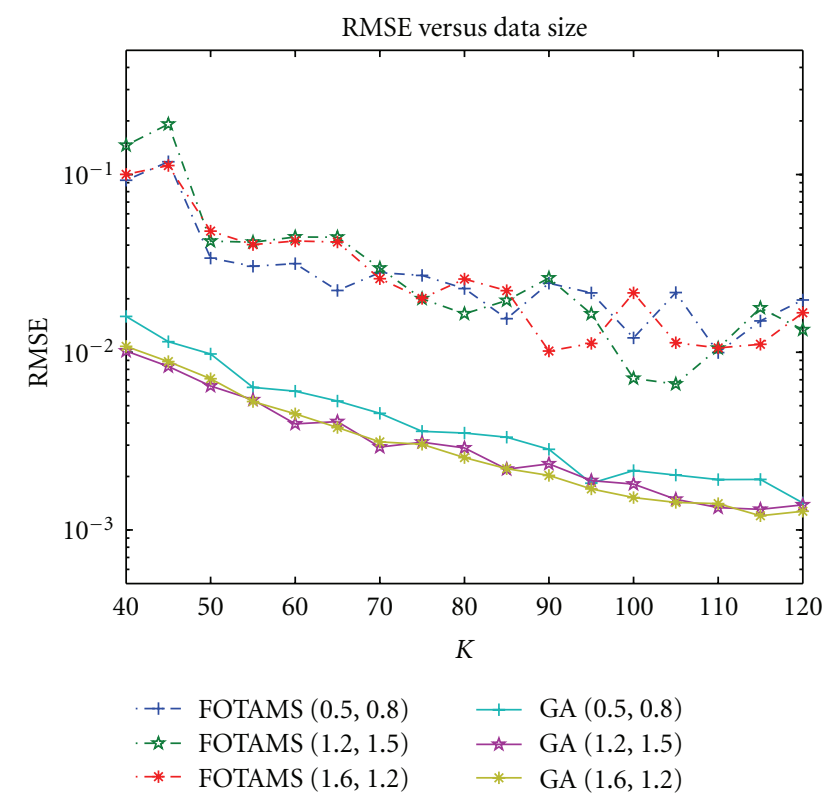

FIGURE 6: RMSEs of frequency estimation versus the data size, $e(m, n)$ is i.i.d white exponential noise, $\mathrm{SNR}=0 \mathrm{~dB}$.

The GA parameters are set as follows. Initial population $M=300$, memorial population $N=120$, genetic times $T=$ 300 , crossover probability $P_{c}=0.6$, mutation probability $P_{m}=0.05$, mutation control parameter $b=1$, niche destining distance $R=0.1$, and individual penalty $P=10^{-4}$.
In this simulation, the performance of the GA-based and the fourth-order time-average moment spectrum-based (FOTAMS) method [13] is compared. Figure 2 shows the root mean squared errors (RMSEs) on the estimated frequency pairs when the data size is $50 \times 50$ which are computed as functions of the SNR from 100 Monte Carlo runs. The frequency estimates of the proposed method are more accurate than that of the FOTAMS-based method whatever the SNR is. The RMSEs of the estimated frequency pairs versus the data size $K \times K$ at $\mathrm{SNR}=0 \mathrm{~dB}$ are shown in Figure 3. It is clear that as the data size increases, the estimation accuracy improves. The estimated values of the proposed method are also more accurate than that of the FOTAMS-based method regardless of the data size.

Example 2. To illustrate that the proposed method is insensitive to the distribution of the noise, $e(m, n)$ is assumed to be the i.i.d. white exponential noise with the mean $m_{e}=0.5$. Other parameters are the same as that in Example 1. Figure 4 shows $\left|\widehat{M}_{4 x}(\alpha, \beta)\right|$ with SNR $=0 \mathrm{~dB}$. Similar to Example 1, we can also observe that three obvious peaks locate at the accurate positions $\left(\omega_{1 l}, \omega_{2 l}\right), l=1,2$, and 3 . Figures 5 and 6 show the RMSEs performance of frequency estimation versus the SNR and the data size, respectively. It is illustrated that the GA estimators also perform better than the FOTAMS estimators.

\section{Conclusion}

In this paper, a cyclic statistics-based method for frequency estimation of $2 \mathrm{D}$ harmonics in correlative multiplicative and additive noise is addressed. Since the $2 \mathrm{D}$ fourth-order time-average moment spectrum peaks at the frequencies of harmonic, the problem of harmonic retrieval can be solved by finding the extremum values. Exploiting global searching ability of GA and the niche phenomenon in nature, we propose a niche GA method to estimate harmonic frequencies. This method can improve the estimation accuracy. Simulation results demonstrated the effectiveness of the presented method. Moreover, our method can be extended to the parameter estimation of $2 \mathrm{D}$ harmonics under other conditions.

\section{Appendix}

\section{Proof of Theorem 1}

$$
\begin{aligned}
& \bar{m}_{4 x}(\tau, \xi) \\
& \triangleq \lim _{T_{1}, T_{2} \rightarrow \infty} \frac{1}{T_{1} T_{2}} \sum_{m=0}^{T_{1}-1} \sum_{n=0}^{T_{2}-1} E\left\{\left(x^{*}(m, n)\right)^{2} x^{*}(m+\tau, n+\xi)\right. \\
& \times x(m+\tau, n+\xi)\}
\end{aligned}
$$




$$
\begin{aligned}
=\lim _{T_{1}, T_{2} \rightarrow \infty} \frac{1}{T_{1} T_{2}} & \\
\times \sum_{m=0}^{T_{1}-1} \sum_{n=0}^{T_{2}-1} E\left\{\sum_{l_{1}=0}^{L} \sum_{l_{2}=0}^{L} \sum_{l_{3}=0}^{L} \sum_{l_{4}=0}^{L} S_{l_{1}}^{*}\right. & (m, n) S_{l_{2}}^{*}(m, n) \\
& \times S_{l_{3}}^{*}(m+\tau, n+\xi) \\
& \times S_{l_{4}}(m+\tau, n+\xi) \\
& \times e^{-j\left(\omega_{1 l_{1}}+\omega_{1 l_{2}}+\omega_{1 l_{3}}-\omega_{1 l_{4}}\right) m} \\
& \times e^{-j\left(\omega_{2 l_{1}}+\omega_{2 l_{2}}+\omega_{2 l_{3}}-\omega_{2 l_{4}}\right) n} \\
& \times e^{-j\left(\omega_{1 l_{3}}-\omega_{1 l_{4}}\right) \tau} \\
& \left.\times e^{-j\left(\omega_{2 l_{3}}-\omega_{2 l_{4}}\right) \xi}\right\}
\end{aligned}
$$$$
=\sum_{l_{1}=0}^{L} \sum_{l_{2}=0}^{L} \sum_{l_{3}=0}^{L} \sum_{l_{4}=0}^{L} E\left\{S_{l_{1}}^{*}(m, n) S_{l_{2}}^{*}(m, n) S_{l_{3}}^{*}(m+\tau, n+\xi)\right.
$$$$
\left.\times S_{l_{4}}(m+\tau, n+\xi)\right\}
$$$$
\times e^{-j\left(\omega_{1 l_{3}}-\omega_{1 l_{4}}\right) \tau} e^{-j\left(\omega_{2 l_{3}}-\omega_{2 l_{4}}\right) \xi}
$$$$
\times \delta\left(-\omega_{1 l_{1}}-\omega_{1 l_{2}}-\omega_{1 l_{3}}+\omega_{1 l_{4}}\right)
$$$$
\times \delta\left(-\omega_{2 l_{1}}-\omega_{2 l_{2}}-\omega_{2 l_{3}}+\omega_{2 l_{4}}\right) \text {. }
$$

According to the formula of Cumulant-Moment (C-M), we have

$$
\begin{gathered}
E\left\{S_{l_{1}}^{*}(m, n) S_{l_{2}}^{*}(m, n) S_{l_{3}}^{*}(m+\tau, n+\xi) S_{l_{4}}(m+\tau, n+\xi)\right\} \\
=E\left\{S_{l_{1}}^{*}(m, n) S_{l_{2}}^{*}(m, n)\right\} \\
\times E\left\{S_{l_{3}}^{*}(m+\tau, n+\xi) S_{l_{4}}(m+\tau, n+\xi)\right\} \\
+\operatorname{Cum}\left\{S_{l_{1}}^{*}(m, n) S_{l_{2}}^{*}(m, n), S_{l_{3}}^{*}(m+\tau, n+\xi)\right. \\
\left.\times S_{l_{4}}(m+\tau, n+\xi)\right\} .
\end{gathered}
$$

In the following, it is proved that the fourth-order timeaverage moment spectrum of the second term (denoted as $M)$ is zero:

$$
\begin{aligned}
\bar{M}=\mid \lim _{T_{1}, T_{2} \rightarrow \infty} \frac{1}{T_{1} T_{2}} & \\
\times \sum_{\tau=0}^{T_{1}-1} \sum_{\xi=0}^{T_{2}-1} \operatorname{Cum}\{ & S_{l_{1}}^{*}(m, n) S_{l_{2}}^{*}(m, n), S_{l_{3}}^{*}(m+\tau, n+\xi) \\
& \left.\times S_{l_{4}}(m+\tau, n+\xi)\right\} e^{-j \alpha \tau} e^{-j \beta \xi} \mid
\end{aligned}
$$

$$
\begin{aligned}
& \leq \lim _{T_{1}, T_{2} \rightarrow \infty} \frac{1}{T_{1} T_{2}} \\
& \times \sum_{\tau=0}^{T_{1}-1} \sum_{\xi=0}^{T_{2}-1} \mid \operatorname{Cum}\left\{S_{l_{1}}^{*}(m, n) S_{l_{2}}^{*}(m, n), S_{l_{3}}^{*}(m+\tau, n+\xi)\right. \\
& \left.\times S_{l_{4}}(m+\tau, n+\xi)\right\} \mid .
\end{aligned}
$$

(Leonov-Shiryaev [14]):

$$
\begin{aligned}
\bar{M} \leq \lim _{T_{1}, T_{2} \rightarrow \infty} \frac{1}{T_{1} T_{2}} & \\
\times \sum_{\tau=0}^{T_{1}-1} \sum_{\xi=0}^{T_{2}-1} \mid & \operatorname{Cum}\left\{S_{l_{1}}^{*}(m, n), S_{l_{3}}^{*}(m+\tau, n+\xi)\right\} \\
& \times \operatorname{Cum}\left\{S_{l_{2}}^{*}(m, n), S_{l_{4}}(m+\tau, n+\xi)\right\} \\
+ & \operatorname{Cum}\left\{S_{l_{1}}^{*}(m, n), S_{l_{4}}^{*}(m+\tau, n+\xi)\right\} \\
\times & \operatorname{Cum}\left\{S_{l_{2}}^{*}(m, n), S_{l_{3}}(m+\tau, n+\xi)\right\} \\
+ & \operatorname{Cum}\left\{S_{l_{1}}^{*}(m, n), S_{l_{2}}^{*}(m, n),\right. \\
& S_{l_{3}}^{*}(m+\tau, n+\xi), \\
& \left.S_{l_{4}}(m+\tau, n+\xi)\right\} \mid .
\end{aligned}
$$

(Triangle Inequality):

$$
\begin{aligned}
\bar{M} \leq \lim _{T_{1}, T_{2} \rightarrow \infty} \frac{1}{T_{1} T_{2}}\{ & \sum_{\tau=0}^{T_{1}-1} \sum_{\xi=0}^{T_{2}-1}\left|C_{S_{l_{1}} S_{l_{3}}}(\tau, \xi) C_{S_{l_{2}} S_{l_{4}}}(\tau, \xi)\right| \\
& +\sum_{\tau=0}^{T_{1}-1} \sum_{\xi=0}^{T_{2}-1}\left|C_{S_{l_{1}} S_{l_{4}}}(\tau, \xi) C_{S_{l_{2}} S_{l_{3}}}(\tau, \xi)\right| \\
& \left.+\sum_{\tau=0}^{T_{1}-1} \sum_{\xi=0}^{T_{2}-1}\left|C_{S_{l_{1}} S_{l_{2}} S_{l_{3}} S_{l_{4}}}(0, \tau, \tau ; 0, \xi, \xi)\right|\right\} .
\end{aligned}
$$

(Schwarz Inequality):

$$
\begin{aligned}
& \bar{M} \leq \lim _{T_{1}, T_{2} \rightarrow \infty} \frac{1}{T_{1} T_{2}} \\
& \times\left\{\sqrt{\sum_{\tau=0}^{T_{1}-1} \sum_{\xi=0}^{T_{2}-1}\left|C_{S_{l_{1}} S_{l_{3}}}(\tau, \xi)\right|^{2} \sum_{\tau=0}^{T_{1}-1} \sum_{\xi=0}^{T_{2}-1}\left|C_{S_{l_{2}} S_{l_{4}}}(\tau, \xi)\right|^{2}}\right. \\
& +\sqrt{\sum_{\tau=0}^{T_{1}-1} \sum_{\xi=0}^{T_{2}-1}\left|C_{S_{l_{1}} S_{l_{4}}}(\tau, \xi)\right|^{2} \sum_{\tau=0}^{T_{1}-1} \sum_{\xi=0}^{T_{2}-1}\left|C_{S_{l_{2}} S_{l_{3}}}(\tau, \xi)\right|^{2}} \\
& \left.+\sum_{\tau=0}^{T_{1}-1} \sum_{\xi=0}^{T_{2}-1}\left|C_{S_{l_{1}} S_{l_{2}} S_{l_{3}} S_{l_{4}}}(0, \tau, \tau ; 0, \xi, \xi)\right|\right\} \text {. }
\end{aligned}
$$


So

$$
\begin{aligned}
\bar{M}_{4 x}(\alpha, \beta) & \\
=\sum_{l_{1}=0}^{L} \sum_{l_{2}=0}^{L} \sum_{l_{3}=0}^{L} \sum_{l_{4}=0}^{L} E & \left\{S_{l_{1}}^{*}(m, n) S_{l_{2}}^{*}(m, n)\right. \\
& \left.\times S_{l_{3}}^{*}(m, n) S_{l_{4}}(m, n)\right\} \\
\times & \delta\left(\alpha+\omega_{1 l_{3}}-\omega_{1 l_{4}}\right) \\
\times & \delta\left(\beta+\omega_{2 l_{3}}-\omega_{2 l_{4}}\right) \\
\times & \delta\left(-\omega_{1 l_{1}}-\omega_{1 l_{2}}-\omega_{1 l_{3}}+\omega_{1 l_{4}}\right) \\
\times & \delta\left(-\omega_{2 l_{1}}-\omega_{2 l_{2}}-\omega_{2 l_{3}}+\omega_{2 l_{4}}\right) .
\end{aligned}
$$

$\bar{M}_{4 x}(\underline{\tau}, \underline{\xi})$ is unequal to zero if and only if

$$
\begin{gathered}
\alpha+\omega_{1 l_{3}}-\omega_{1 l_{4}}=0 \bmod (2 \pi), \\
\beta+\omega_{2 l_{3}}-\omega_{2 l_{4}}=0 \bmod (2 \pi), \\
\omega_{1 l_{1}}+\omega_{1 l_{2}}+\omega_{1 l_{3}}=\omega_{1 l_{4}} \bmod (2 \pi), \\
\omega_{2 l_{1}}+\omega_{2 l_{2}}+\omega_{2 l_{3}}=\omega_{2 l_{4}} \bmod (2 \pi) .
\end{gathered}
$$

We analyze four cases as follows.

(1) None of the four frequency pairs satisfying (A.9) and (A.10) are unequal to $(0,0)$. According to (AS1), it is impossible.

(2) Only one of the four frequency pairs satisfying (A.9) and (A.10) is zero. According to (AS1), it is impossible.

(3) Two of the four frequency pairs satisfying (A.9) and (A.10) are equal to $(0,0)$. Since $\left(\omega_{1 l_{4}}, \omega_{2 l_{4}}\right)$ is not $(0,0)$ according to (AS1), there must be two pairs equal to $(0,0)$ among $\left(\omega_{1 l_{1}}, \omega_{2 l_{1}}\right),\left(\omega_{1 l_{2}}, \omega_{2 l_{2}}\right)$, and $\left(\omega_{1 l_{3}}, \omega_{2 l_{3}}\right)$.

Thus, the fourth-order time-average moment spectrum is

$$
\begin{aligned}
& \bar{M}_{4 x}(\alpha, \beta) \\
&=\sum_{l_{3}=1}^{L} E\left\{S_{0}^{*}(m, n) S_{0}^{*}(m, n)\right\} E\left\{S_{l_{3}}^{*}(m, n) S_{l_{3}}(m, n)\right\} \\
& \times \delta(\alpha) \delta(\beta) \\
&+\sum_{l_{2}=1}^{L} E\left\{S_{0}^{*}(m, n) S_{l_{2}}^{*}(m, n)\right\} E\left\{S_{0}^{*}(m, n) S_{l_{2}}(m, n)\right\} \\
& \times \delta\left(\alpha-\omega_{1 l_{2}}\right) \delta\left(\beta-\omega_{2 l_{2}}\right) \\
&+\sum_{l_{1}=1}^{L} E\left\{S_{l_{1}}^{*}(m, n) S_{0}^{*}(m, n)\right\} E\left\{S_{0}^{*}(m, n) S_{l_{1}}(m, n)\right\} \\
& \times \delta\left(\alpha-\omega_{1 l_{1}}\right) \delta\left(\beta-\omega_{2 l_{1}}\right) \\
&=2 \sum_{l_{1}=1}^{L} E\left\{S_{0}^{*}(m, n) S_{l_{1}}^{*}(m, n)\right\} E\left\{S_{0}^{*}(m, n) S_{l_{1}}(m, n)\right\} \\
& \times \delta\left(\alpha-\omega_{1 l_{1}}\right) \delta\left(\beta-\omega_{2 l_{1}}\right) \\
&+\sum_{l_{2}=1}^{L} E\left\{S_{0}^{*}(m, n) S_{0}^{*}(m, n)\right\} E\left\{S_{l_{2}}^{*}(m, n) S_{l_{2}}(m, n)\right\} \\
& \times \delta(\alpha) \delta(\beta) .
\end{aligned}
$$

(4) All of the four frequency pairs satisfying (A.9) and (A.10) are unequal to $(0,0)$. Thus, the fourth-order time-average moment spectrum is

$$
\begin{aligned}
\bar{M}_{4 x}(\alpha, \beta)= & E\left\{S_{0}^{*}(m, n) S_{0}^{*}(m, n)\right\} \\
& \times E\left\{S_{0}^{*}(m, n) S_{0}(m, n)\right\} \delta(\alpha) \delta(\beta),
\end{aligned}
$$

thus yielding

$$
\begin{aligned}
& \bar{M}_{4 x}(\alpha, \beta) \\
& =2 \sum_{l_{1}=1}^{L} E\left\{s_{0}^{*}(m, n) s_{l_{1}}^{*}(m, n)\right\} E\left\{s_{0}^{*}(m, n) s_{l_{1}}(m, n)\right\} \\
& \quad \times \delta\left(\alpha-\omega_{1 l_{1}}\right) \delta\left(\beta-\omega_{2 l_{1}}\right) \\
& +\left[\sum_{l_{2}=1}^{L} E\left\{s_{0}^{*}(m, n) s_{0}^{*}(m, n)\right\} E\left\{s_{l_{2}}^{*}(m, n) s_{l_{2}}(m, n)\right\}\right. \\
& \left.\quad+E\left\{s_{0}^{*}(m, n) s_{0}^{*}(m, n)\right\} E\left\{s_{0}^{*}(m, n) s_{0}(m, n)\right\}\right] \delta(\alpha) \delta(\beta) .
\end{aligned}
$$

\section{Acknowledgments}

The authors would like to thank the anonymous reviewers for their constructive comments and suggestions that helped to improve the paper. This work is supported by the National Natural Science Foundation of China under Grants nos. 60736009 and 60901066.

\section{References}

[1] J. W. Odendaal, E. Barnard, and C. W. I. Pistorius, "Twodimensional superresolution radar imaging using the MUSIC algorithm," IEEE Transactions on Antennas and Propagation, vol. 42, no. 10, pp. 1386-1391, 1994.

[2] Y. Hua, "Estimating two-dimensional frequencies by matrix enhancement and matrix pencil," IEEE Transactions on Signal Processing, vol. 40, no. 9, pp. 2267-2280, 1992.

[3] S. Rouquette and M. Najim, "Estimation of frequencies and damping factors by two-dimensional ESPRIT type methods," IEEE Transactions on Signal Processing, vol. 49, no. 1, pp. 237245, 2001.

[4] H. M. Ibrahim and R. R. Gharieb, "Estimating twodimensional frequencies by a cumulant-based FBLP method," IEEE Transactions on Signal Processing, vol. 47, no. 1, pp. 262266, 1999.

[5] R. R. Gharieb, "Cumulant-based LP method for twodimensional spectral estimation," IEEE Proceedings: Vision, Image and Signal Processing, vol. 146, no. 6, pp. 307-312, 1999.

[6] G. B. Giannakis and G. Zhou, "Harmonics in multiplicative and additive noise: parameter estimation using cyclic statistics," IEEE Transactions on Signal Processing, vol. 43, no. 9, pp. 2217-2221, 1995.

[7] R. F. Dwyer, "Fourth-order spectra of Gaussian amplitudemodulated sinusoids," Journal of the Acoustical Society of America, vol. 90, no. 2, pp. 918-926, 1991.

[8] O. Besson and P. Stoica, "Sinusoidal signals with random amplitude: least-squares estimators and their statistical analysis," IEEE Transactions on Signal Processing, vol. 43, no. 11, pp. 2733-2744, 1995. 
[9] F. Wang, S.-X. Wang, and H.-J. Dou, "Two-dimensional parameter estimation using two-dimensional cyclic statistics," Journal of Electronics, vol. 31, no. 10, pp. 1522-1525, 2003 (Chinese).

[10] S.-Y. Yang and H.-W. Li, "Two-dimensional harmonics parameters estimation using third-order cyclic-moments," Journal of Electronics, vol. 33, no. 10, pp. 1808-1811, 2005 (Chinese).

[11] S. Wu and H. Li, "The analysis of two-dimensional quadratic coupled harmonics in the complex noise based on genetic algorithm," Signal Processing Journal, vol. 22, pp. 635-638, 2006 (Chinese).

[12] J. Xu, S.-X. Wang, and H. Wang, "Harmonic retrieval and nonlinear frequency-coupled harmonics in the complex noise," Journal of Electronics, vol. 31, pp. 117-122, 2003 (Chinese).

[13] H. Dou, S.-X. Wang, and F. Wang, "Two-dimensional harmonics retrieval in correlative multiplication and additive noise," in Proceedings of the 7th International Conference on Signal Processing (ICSP '04), pp. 264-268, Beijing, China, September 2004.

[14] D. R. Brillinger, Time Series: Data Analysis and Theory, Holden-day, San Francisco, Calif, USA, 1981.

[15] A. V. Dandawate and G. B. Giannakis, "Asymptotic theory of mixed time averages and kth-order cyclic-moment and cumulant statistics," IEEE Transactions on Information Theory, vol. 41, no. 1, pp. 216-232, 1995.

[16] H. Li, Q. Cheng, and B. Yuan, "Strong laws of large numbers for two-dimensional processes," in Proceedings of the 4th International Conference on Signal Processing (ICSP '98), pp. 43-46, Beijing, China, October 1998.

[17] D. E. Goldberg, Genetic Algorithms in Search, Optimization and Machine Learning, Addison-Wesley, Reading, Mass, USA, 1989.

[18] Z. Michalewicz, Genetic Algorithms + Data Structures = Evolution Programs, Springer, New York, NY, USA, 3rd edition, 1999.

[19] K. Deb and D. E. Coldberg, "An investigation of niche and species formation in genetic function optimization," in Proceedings of the 3 rd Conference on Genetic Algorithms (ICGA '89), pp. 42-50, Morgan Kaufmann, San Mateo, Calif, USA, 1989.

[20] M. Zhou and S. Sun, The Theory and Application of Genetic Algorithm, The Press of National Defence and Industry, Beijing, China, 1999.

[21] S. Yang and H. Li, "Estimation of the number of harmonics in multiplicative and additive noise," Signal Processing, vol. 87, no. 5, pp. 1128-1137, 2007.

[22] S. Yang and H. Li, "Estimating the number of harmonics using enhanced matrix," IEEE Signal Processing Letters, vol. 14, no. 2, pp. 137-140, 2007. 